\title{
Intervenção psicológica em adultos obesos com o transtorno da compulsão alimentar periódica (TCAP)
}

\author{
Psychological intervention in obese adults with periodic binge eating \\ disorder (BED) \\ Intervención psicológica em adultos obesos com El trastorno de La \\ compulsión alimentaria periódica (TCAP) \\ Tatiane Aparecida Enside de Abreu MARÇAL \\ Elerson Gaetti JARDIM JÚNIOR
}

Faculdade de Odontologia de Araçatuba

Univ Estadual Paulista, UNESP 16015.050 Araçatuba-SP, Brasil

\begin{abstract}
Resumo
A obesidade é considerada, tanto em países em desenvolvimento quanto em países desenvolvidos, um importante problema de saúde pública,e, de acordo com a Organização Mundial da Saúde (OMS), há mais de um bilhão de adultos com excesso de peso e pelo menos trezentos milhões são obesos.Associado à obesidade existe o transtorno da compulsão alimentar, o qual é caracterizado por um distúrbio persistente da alimentação ou comportamento relacionado com a alimentação, resultando em alterações no consumo ou na absorção do alimento. O presente estudo se propôs a identificar na literatura os principais aspectos do diagnóstico dos pacientes acometidos pelo transtorno da compulsão alimentar periódica (TCAP), apontar os impactos que este transtorno pode ter na qualidade emocional e de vida, bem como enfatizar a importância da terapia cognitivo-comportamental (TCC) como forma de tratamento para estes pacientes. Foram pesquisados nas bases de dados: SciELO (Scientific Electronic Library Online), CAPES, Embase, PubMed (National Library ofMedicine), MEDLINE e DECS artigos que tratassem da obesidade, do transtorno da compulsão alimentar em adultos e da terapia Cognitivo-Comportamental. Observou-se que o transtorno da compulsão alimentar periódica deve ser tratado como uma categoria distinta de transtorno alimentar, conforme o Diagnostic and Statistical Manual of Mental Disorders, atualmente em sua quinta edição, e a terapia cognitiva comportamental vem mostrando eficácia, proporcionando uma redução significativa da compulsão alimentar na maioria dos pacientes.
\end{abstract}

Descritores: Obesidade; Transtorno da Compulsão Alimentar; Terapia Cognitiva.

\section{Abstract}

Obesity is considered a major public health problem in both developing and developed countries and, according to the World Health Organization (WHO), there are over one billion overweight and at least three hundred million are obese. Associated with obesity there is binge eating disorder, which is characterized by a persistent eating disorder or related eating behavior, resulting in changes in food intake or absorption. The present study aimed to identify in the literature the main aspects of the diagnosis of patients with periodic binge eating disorder (BED), to point out the impacts that this disorder may have on emotional and life quality, as well as to emphasize the importance of cognitive therapy (CBT) as a form of treatment for these patients. We searched the databases (ScientificElectronic Library Online), CAPES, Embase, PubMed (National Library of Medicine), MEDLINE and DECS articles dealing with obesity, binge eating disorder in adults and Cognitive-Behavioral therapy. It was observed that periodic binge eating disorder should be treated as a distinct category of eating disorder, according to the Diagnostic and Statistical Manual of Mental Disorders, currently in its fifth edition, and cognitive behavioral therapy has shown efficacy, providing a significant reduction of binge eating in most patients.

Descriptors: Obesity; Binge-Eating Disorder; Cognitive Therapy.

\section{Resumen}

La obesidad se considera, tanto en países en desarrollo como en países desarrollados, un importante problema de salud pública, y, según la Organización Mundial de la Salud (OMS), hay más de mil millones de adultos con sobrepeso y al menos trescientos millones son obesos. En el caso de la obesidad existe el trastorno de la compulsión alimentaria, el cual se caracteriza por un disturbio persistente de la alimentación o comportamiento relacionado con la alimentación, resultando en alteraciones en el consumo o en la absorción del alimento. El presente estudio se propuso identificar en la literatura los principales aspectos del diagnóstico de los pacientes acometidos por el trastorno de la compulsión alimentaria periódica (TCAP), señalar los impactos que este trastorno puede tener en la calidad emocional y de vida, así como enfatizar la importancia de la terapia cognitiva -comportamental (TCC) como forma de tratamiento para estos pacientes. En las bases de datos aSciELO (ScientificElectronic Library Online), CAPES, Embase, PubMed (National Library ofMedicine), MEDLINE y DECS artículos que tratase de la obesidad, del trastorno de la compulsión alimentaria en adultos y de la terapia Cognitivo-Comportamental. Se observó que el trastorno de la compulsión alimentaria periódica debe ser tratado como una categoría distinta de trastorno alimentario, según el Diagnostic and Statistical Manual of Mental Disorders, actualmente en su quinta edición, y la terapia cognitiva conductual viene mostrando eficacia, proporcionando una reducción significativa de la compulsión alimentaria en la mayoría de los pacientes.

Descriptores: Obesidad; Trastorno Por Atrácon; Terapia Cognitiva.

\section{INTRODUÇÃO}

Recentemente definido, o Transtorno da Compulsão Alimentar Periódica (TCAP) é uma categoria diagnóstica geralmente associada com psicopatologia e excesso de peso, podendo acarretar obesidade. Sua prevalência é de $0,7 \%$ a $4 \%$ na população em geral, $30 \%$ na população de obesos em tratamento e $50 \%$ na população de obesos graves ${ }^{1}$.Nesse sentido, estudos da morbidade e do "sobrepeso" apontam que os transtornos psiquiátricos serão um dos principais problemas de saúde pública no século ${ }^{1}$.

Segundo a Organização Mundial da Saúde (OMS), há mais de um bilhão de adultos com excesso de peso e pelo menos trezentos milhões são obesos, de forma que a obesidade constitui sério problema de saúde pública no mundo todo, uma verdadeira epidemia silenciosa global, tanto em países economicamente desenvolvidos quanto naqueles que se encontram em vias de desenvolvimento ${ }^{2}$.

Essa epidemia se apresenta como uma condição multifatorial, mas merece destaque sua relação com os distúrbios alimentares, que são condições severas que ocorrem em diferentes faixas etárias e em ambos os $\operatorname{sexos}^{3} \mathrm{e}$ estão associados à compulsão alimentar, caracterizada pela ingestão de grande quantidade de alimentos em periodos curtos de tempo, e uma sensação de perda de controle sobre o "comer" 4 - 6 .

Esses Transtornos Alimentares (TA) estão associados a duas categorias diagnósticas reconhecidas pelo "Diagnostic and Statistical Manual of Mental Disorder" (DSM) da Associação Psiquiátrica Norte-americana e pela Classificação Estatística Internacional de Doenças e Problemas Relacionados à Saúde (CID) da OMS: Anorexia Nervosa 
(AN) e Bulimia Nervosa $(\mathrm{BN})^{7,8}$.Como agravante, a literatura sobre esses transtornos é particularmente voltada para os países do hemisfério norte, notadamente os Estados Unidos e países europeus ${ }^{9-11}$, muito ainda há para se discutir sobre esses transtornos alimentares, que a cada dia vem acometendo pessoas das mais diversas idades.

Dentre as abordagens terapêuticas desses transtornos, em função de seus componentes comportamentais, destaca-se a terapia cognitivo-comportamental, que consiste em um tipo de tratamento psicoterápico no qual auxilia os pacientes a compreenderem os seus pensamentos e sentimentos os quais afetam a maneira de se comportar.

Embora a epidemiologia da obesidade ligada aos transtornos alimentares evidencie uma base comportamental, onde atuariam os diferentes componentes ligados à vida pessoal e comunitária dos afetados, poucos são os estudos que avaliam o impacto da Terapia Cognitivo-Comportamental (TCC) no tratamento e acompanhamento desses pacientes.

Dessa forma, o presente estudo se propôs a identificar, por meio de revisão de literatura, os principais aspectos do diagnóstico dos pacientes acometidos pelo TCAP, apontar os impactos que este transtorno pode ter na qualidade emocional e de vida dosacometidos, bem como enfatizar a importância da Terapia Cognitivo-Comportamental (TCC) como forma de tratamento para esses distúrbios

\section{MATERIAL E MÉTODO}

O presente estudo consistiu em pesquisa de avaliação descritiva da literatura científica, por meio do serviço de rede VPN (virtual private network), empregando-se o sistema de busca "Google Acadêmico" com as seguintes palavraschave:binge, binge eating, periodic binge eating desorder, obesity, cognitive behavior therapy, cognitive therapy, obesidade, transtorno da compulsão alimentar periódica e terapia cognitiva comportamental.

As bases de dados indexadas e consultadas foram SciELO (ScientificElectronic Library Online), CAPES, Embase, PubMed (National Library of Medicine), MEDLINE e DECS. Foram incluídos na pesquisa apenas os estudos relacionados com o transtorno da compulsão alimentar periódica em pacientes obesos adultos e a intervenção psicológica como forma de tratamento. Foram excluídos os estudos cuja intervenção psicológica no tratamento desses pacientes não incluía ou priorizava a terapia cognitivocomportamental, além de artigos associando a TCC à farmacoterapia, artigos informativos, manuais de auto ajuda ou com finalidade de divulgação/promocional, bem como textos que apenas descreviam casos clínicos ou associados à obesidade infantil ou intervenções terapêuticas em crianças.

\section{RESULTADOS E DISCUSSÃO}

\section{- Obesidade no Brasil e no mundo}

A obesidade é um importante problema de saúde publica global. A má alimentação vem crescendo e ganhando espaço, potencializada pelo apelo comercial e propagandístico dos produtos industrializados e "fastfoods", o que muito contribui para converter a obesidade em sério problema de saúde no Brasil e no resto do mundo ${ }^{12}$.

Um exemplo bastante evidente e dramático do quadro pode ser visto nos Estados Unidos da América do Norte, onde a obesidade aumentou em mais de $75 \%$ desde $1980^{13}$ Dados da OMS indicam que 54\% dos adultosnorte-americanos apresentam sobrepeso e $22 \%$ são obesos ${ }^{14,15}$, enquanto que pesquisas epidemiológicas apontam que o aumento de de $2 \%$ ao ano observado para a obesidade, nas últimas décadas, deverá persistir nos próximos anos ${ }^{14}$. Caso esta tendência não seja interrompida, é possível estimar que praticamente toda a população norte-americana apresentará sobrepeso em algumas gerações futuras ${ }^{14-17}$.

No Brasil, o sobrepeso já afeta mais da metade da população e $20 \%$ dos adultos estão obesos, de acordo com o relatório das Nações Unidas para Alimentação e Agricultura $^{18}$. A proporção de adultos com sobrepeso no Brasil aumentou de 51\% em 2010, para 54\% em 2014, sendo que $17 \%$ dos homens e $23 \%$ das mulheres estão obesas e esse fenômeno não dá sinal de arrefecimento e deve se agravar ainda mais.

$\mathrm{Na}$ Europa, verificou-se em uma década um crescimento entre $10 \%$ a $40 \%$ da obesidade na maior parte dos países. Na região Oeste do Pacífico, abrangendo a Austrália, o Japão, Samoa e China, também se observa o incremento da obesidade. Entretanto, China e Japão, mesmo com o aumento da obesidade em relação a outros países desenvolvidos, possuem as menores prevalências mundiais. Nos continentes africano e asiático, a obesidade é ainda relativamente incomum, sendo sua prevalência mais elevada na população urbana. Porém, nas regiões economicamente desenvolvidas destes continentes, a ocorrência da obesidade pode ser tão alta quanto nos países desenvolvidos ${ }^{19}$.

\section{- Obesidade: Diagnóstico}

A preocupação com a obesidade tem assumido destaque no cenário internacional e nacional, haja vista os inúmeros fóruns e resoluções emanados dos principais órgãos de regulação da saúde, como a OMS e instituições administrativas, como o National Instituteof Health (NIH) nos Estados Unidos e o Ministério da Saúde do Brasil ${ }^{20}$.

Dentre as várias formas de mensuração da obesidade, o Índice de Massa Corpórea (IMC), tornou-se o padrão referencial para essa avaliação, tanto em âmbito individual como populacional $^{21-23}$.

O IMC é um padrão internacional de cálculo da obesidade, parâmetro bastante utilizado para classificar o indivíduo de acordo com seu peso e altura. Seu uso é disseminado principalmente entre os profissionais que trabalham com o corpo. Dada sua praticidade, simplicidade e elevado grau de confiabilidade na indicação da gordura corporal, correlacionando-se inclusive com medidas diretas, o IMC permitiu a classificação da obesidade em quatro níveis graus I, II, III (grave ou mórbida) e super-obesidade, constituindo-se no mais importante índice para fim de diagnóstico e categorização, dentre vários outros parâmetros antropométricos e clínico-laboratoriais indicativos de obesidade e risco para doenças associadas ${ }^{22,24}$.

A OMS indica a antropometria como método para diagnóstico da obesidade,pois é o mais barato, não-invasivo, universalmente aplicável e com boa aceitação pelapopulação ${ }^{25,26}$. De acordo com a adaptação adotada pela OMS $^{27}$, apresentada na Tabela 1, o diagnóstico baseia-se no IMC, em padrões internacionais desenvolvidos para pessoas adultas descendentes de europeus, o que traz alguns questionamentos, uma vez que diferentes grupos étnicoraciais apresentam padrões físicos bastante diversos.

Tabela 1. Classificação de peso pelo IMC

\begin{tabular}{ccc} 
Classificação & $\mathbf{I M C}\left(\mathbf{k g} / \mathbf{m}^{\mathbf{2}}\right)$ & Risco de comorbidades \\
\hline Baixo Peso & $<18,5$ & Baixo \\
Peso normal & $18,5-24,9$ & Médio \\
Sobrepeso & $\geq 25$ & - \\
Pré-obeso & 25,0 a 29,9 & Aumentado \\
Obeso I & 30,0 a 34,9 & Moderado \\
Obeso II & 35,0 a 39,9 & Grave \\
Obeso III & $\geq 40,0$ & Muito Grave
\end{tabular}

- Transtorno da Compulsão Alimentar Periódica (TCAP)

A compulsão alimentar é caracterizada por um distúrbio persistente da alimentação ou por comportamento relacionado com a alimentação, resultando em alterações no consumo ou na absorção do alimento. Dentre os critérios de diagnósticos, tem-se a anorexia nervosa, bulimia nervosa e o 
transtorno da compulsão alimentar periódica (TCAP) ${ }^{7}$.

Observa-se que os indivíduos com TCAP apresentam autoestima mais baixa ${ }^{28}$ e se importam mais com o peso e a forma corporal do que outros pacientes obesos que não possuem o transtorno ${ }^{29}$. Destaca-se que o transtorno da compulsão alimentar também é seguido por sensações de ansiedade subjetiva, somando-se com constrangimento, repugnância e/ou culpa. Estudos apontam que um comedor compulsivo engloba no mínimo dois elementos: o subjetivo, em que é observado o sentimento de falta de controle, e o objetivo, relacionado com a quantidade do consumo alimentar $^{30}$. Além disso, o estresse é um elemento o qual pode conduzir ao incremento das compulsões alimentares. Em períodos de estresse, o cortisol é liberado estimulando a ingestão de alimentos e o aumento do peso ${ }^{30,31}$.

Este artigo dará enfoque ao diagnóstico da compulsão alimentar periódica, descrito a seguir conforme o DSM- $\mathrm{V}^{7}$ :

A. Um episódio da compulsão alimentar é caracterizado por ambas as situações que seguem:

1. Alimentação, em um curto período de tempo (ou seja, período em torno de duas horas), com uma quantidade de comida que é definitivamente maior do que a maioria das pessoas poderia comer em um período similar de tempo sob as mesmas circunstâncias.

2. Uma sensação de perda de controle da saciedade (ou seja, um sentimento de não poder parar ou controlar o que se come e ou quanto se está comendo).

B. Os episódios de compulsão alimentar são associados com três (ou mais) das seguintes situações:

1. Ingerir alimento muito mais rapidamente do que o normal.

2. Ingerir alimento até se sentir inconfortavelmente saciado.

3. Ingerir grandes quantidades de comida quando não estiver com fome.

4. Ingerir alimento sozinho para esconder a voracidade

5. Sentir-se desgostoso, depressivo ou muito culpado depois de comer.

C. Profundo mal-estar marcado em relação à compulsão alimentar.

D. Ocorrência de compulsão alimentar, em média, pelo menos uma vez na semana por 3 meses.

E. A ingestão compulsiva não se associa a estratégias compensatórias inadequadas (por exemplo, purgantes, jejum, exercício físico excessivo) e não ocorre exclusivamente durante o decurso de anorexia nervosa ou bulimia nervosa.

Dessa forma é importante destacar que os critérios de diagnósticos são a melhor forma de avaliar como os transtornos mentais são expressados e como podem ser reconhecidos nos tratamentos clínicos podendo, portanto, ajudar no diagnóstico e tratamento desse transtorno.

- Intervenção Psicológica através da Terapia Cognitiva Comportamental (TCC), como forma de tratamento do TCAP

A Terapia Cognitivo-Comportamental (TCC) pode ser definida como um método terapêutico para tratar problemas psicológicos e que auxilia os pacientes a compreenderem o sistema mental e emoções que afetam comportamentos. A terapia cognitivo-comportamental também pode ser definida como uma intervenção semi estruturada, objetiva e orientada para metas, que aborda fatores cognitivos, emocionais e comportamentais no tratamento dos transtornos psiquiátricos $^{32}$. A intervenção psicológica por meio da TCC é comumente utilizada para tratar uma ampla gama de distúrbios, incluindo fobias, vícios, depressão e ansiedade.

Reconhecida como o método de terapia para tratar problemas psicológicos mais pesquisados para o tratamento do TCAP, a terapia cognitivo-comportamental tem sido amplamente abordada em vários locais especializados em transtornos mentais ${ }^{33-38}$ e se fundamenta na hipótese de que um complexo distúrbio de princípios está relacionado à manifestação e permanência do TCAP. Em virtude disto, os cernes do tratamento são a alteração de padrões distorcidos de pensamentos e a reestruturação de crenças supervalorizadas relacionadas ao peso e à imagem do corpo, sendo abordadas diversas técnicas cognitivas com esse intuito.

Tem se demonstrado de grande importância para o tratamento de indivíduos com TCAP, apresentando características específicas como a estrutura, brevidade, orientação para o presente, direcionamento para resolução de problemas e modificação de pensamentos e comportamentos disfuncionais ${ }^{39}$.Especificamente em relação à TCC para o emagrecimento, parte-se do pressuposto que modificando o pensamento, o comportamento alimentar também será alterado e desta forma se dará a perda de peso ${ }^{40,41}$.

A TCC convencional é em geral um tratamento de curto prazo (cerca de 12 a 16 sessões) que enfoca aspectos cognitivos do problema (pensamentos distorcidos) tais como a auto-avaliação centrada no peso e forma do corpo, baixa auto-estima, perfeccionismo e outros aspectos ${ }^{42}$.

Além de técnicas cognitivas, a TCC também emprega técnicas comportamentais para ajudar na modificação dos hábitos alimentares. Como exemplos dessas técnicas, podem ser citadas:

- a auto monitoração, com a observação sistemática e registro dos alimentos ingeridos e das circunstâncias associadas;

- as técnicas para controle de estímulos, que envolvem a identificação das situações que favorecem a ocorrência da compulsão alimentar e o desenvolvimento de um estilo de vida que minimize o contato do paciente com essas situações;

- treinamento em resolução de problemas, que ajuda o paciente a desenvolver estratégias alternativas para enfrentar suas dificuldades sem recorrer à alimentação inadequada.

Em geral, a TCC também focaliza estratégias para prevenção de recaídas ${ }^{43-45}$.

No tratamento psicoterápico, a terapia cognitiva vem mostrando eficácia por trabalhar a partir da estrutura operante do paciente com objetivos de organizar as contingências para mudanças de peso e comportamentos, em princípio, relacionados ao autocontrole de comportamentos alimentares, e contexto situacional amplo, aprofundando para todo o desconforto $^{46,47}$. A avaliação e correção dos pensamentos inadequados, que contribuem tanto para a etiologia quanto para a manutenção da obesidade, são procedimentos disparadores e frequentes no processo psicoterapêutico para a modificação comportamental. A reestruturação cognitiva, imagens orientadas, o treinamento da autoinstrução, a determinação de objetivos, o estímulo ao autor reforço e resolução de problemas são alguns procedimentos interrelacionados, de base cognitiva, incorporados a outros programas comportamentais ${ }^{46,47}$.

Nesse sentido, conclui-se que o transtorno da compulsão alimentar periódica deve ser tratado como uma categoria distinta de transtorno alimentar, conforme o DSM$\mathrm{V}$, e a terapia cognitiva comportamental vem mostrando eficácia por objetivar a organização das contingências para mudanças de peso e comportamentos, em princípio, relacionados ao autocontrole de comportamentos alimentares e contexto situacional mais amplo, e tem sua eficácia demonstrada no tratamento do $\mathrm{TCAP}^{48}$, proporcionando uma redução significativa da compulsão alimentar na maioria dos pacientes.

\section{CONCLUSÃO}

Observou-se que o indivíduo acometido pelo transtorno da compulsão alimentar periódica, quando submetido ao tratamento psicoterápico da terapia cognitivo comportamental, obtém mudanças significativas nos 
pensamentos e comportamentos disfuncionais relacionados à supervalorização da imagem e do peso por meio de técnicas cognitivas, alterando desta forma o comportamento alimentar e consequentemente auxiliando a perda de peso.

Com o crescimento de estudos nesta área da psicoterapia cognitivo comportamental será possível no futuro oferecer suporte adequado a este grupo de indivíduos acometidos por transtornos psiquiátricos, os quais serão um dos principais problemas de saúde pública no século 21 .

\section{REFERÊNCIAS}

1. Hay PJ. Epidemiologia dos transtornos alimentares: estado atual e desenvolvimentos futuros. Rev Bras Psiquiatr. 2002; 24(Sup3):13-7.

2. Organização Pan-Americana da Saúde. Doenças crônicodegenerativas e obesidade: estratégia mundial sobre alimentação saudável atividade física e saúde. Brasília: OPS; 2003

3. Palavras MA, Hay P, Santos Filho CA, Claudino A. The efficacy of psychological therapies in reducing weight and binge eating in people with bulimia nervosa and binge eating disorder who are overweight or obese: a critical synthesis and meta-analyses. Nutrients. 2017; 9(3):E299.

4. Borges MB, Jorge MR, Morgan CM, Da Silveira DX, Custódio O. Binge-eating disorder in Brazilian women on a weight-loss program. Obes Res. 2002; 10(11):1127-34.

5. Quintero-Párraga E, Pérez-Montiel AC, Montiel-Nava C, Pirela D, Acosta MF, Pineda N. Trastornos de laconducta alimentaria: prevalência y características clínicas em adolescentes de laciudad de Maracaíbo, Estado Zulia, Venezuela. Invest Clin. 2003; 44(3):179-93.

6. Brandão PP, Garcia-Souza EP, Neves FA, Pereira MJ, Sichieri R, Moura EG, et al. Leptin/adiponectin ratio in obese women with and without binge eating disorder. Neuro Endocrinol Lett. 2010; 31(3):353-8.

7. American Psychiatric Association. Manual diagnóstico e estatístico de transtornos mentais: DSM-5. 5th ed. Porto Alegre: Artmed; 2014.

8. Organização Mundial da Saúde. Classificação de transtornos mentais e de comportamento da CID 10:descrições clínicas e diretrizes diagnóstica. Porto Alegre: Artes Médicas; 1993.

9. Stunkard AJ. Eating patterns and obesity. Psychiatr Q 1959; 33:284-95.

10. Wermuth BM, Davis KL, Hollister LE, Stunkard AJ. Phenytoin treatmentof the binge eating syndrome. Am J Psychiatry. 1977; 134(11):1249-53.

11. Myers LL, Wiman AM. Binge eating disorder: a review of a new "DSM" diagnosis. Res Soc Work Pract. 2014; 24(1):86-95.

12. LeRoith D, Karnieli E. Obesity. Med Clin North Am. 2011; 95(5):855-1040.

13. Yanovski SZ, Yanovski JA. Obesity. N Engl J Med. 2002; 346(8):591-602.

14. Lerario AC, Lottenberg SA. Mecanismos ambientais implicados no ganho de peso e as oportunidades para prevenção da obesidade. Einstein. 2006; 4(Supl 1):S7-13.

15. Flegal MG, Carroll MD, Kuczmarszaki RJ, Johnson CL. Overweight and obesity in the United States: prevalence and trends, 1960-1994. Int J Obes Relat Metab Disord. 1998; 22(1):39-47.

16. Hill JO, Peters JC. Environmental contributions to the obesity epidemic. Science. 1998; 280(5368):1371-4.

17. Poston WS 2nd, Foreyt JP. Obesity is an environmental issue. Atherosclerosis. 1999; 146(2):201-9.

18. World Health Organization. Sobrepeso e obesidade aumentam no Brasil segundo relatório da FAO e OPAS. 2017. Disponível em: http://www.fao.org/ americas/noticias/ver/ pt/c/466066. Acesso em: $10 \mathrm{de}$ outubro de 2017.

19. World Health Organization. Obesity: Preventing and managing the global epidemic: report of a WHO consultation on obesity. Geneva: WHO; 1998.

20. Brasil. Ministério da Saúde. Secretaria de Assistência à Saúde. Portaria $n^{\circ}$ 492, de 31 de agosto de 2007. Diário Oficial da União. 2007; 31 ago.

21. Keil U, Kuulasmaa K. WHO MONICA Project risk factors. Int J Epidemiol. 1989; 18(Suppl 1):S46-55.

22. Santo MA, Cecconello I. Morbid obesity: risks control. Arq Gastroenterol. 2008; 45(1):1-2.

23. Quetelet A. Physique sociale: ou, essai sur le développement des Facultés de L’Homme. Brussels: C. Muquardt; 1869.

24. Garrido Chamorro RP, González Lorenzo M. Índice de masa corporal y composición corporal: um estudio antropométrico de 2500 deportistas de alto nivel. Efedeportes. 2004; 10(76). Disponível em: http://www.efdeportes.com/efd76/antrop.htm. Acesso em: 10 de outubro de 2017.

25. World Health Organization. Physical status: the use and interpretation of anthropometry. Geneva: WHO; 1995.

26. Kweitel S. IMC: herramienta poco útil para determinar el peso útil de un deportista. Rev Int Med Ciênc Act Fís Deporte. 2007; 7(28):274-89.

27. World Health Organization. Obesity: preventing and managing the global epidemic: report of a World Health Organization Consultation. Geneva: WHO; 2000.

28. de Zwaan M, Mitchell JE, Seim H, Specker SM, Pyle RL, Raymond N, et al. Eating related and general psychopathology in obese females with binge eating disorder. Int J Eat Dis. 1994; 15(1):43-52.

29. Wilson GT, Nonas CA, Rosenblum GD. Assessment of binge eating in obese patients. Int J Eat Disord. 1993; 13(1):25-33.

30. Azevedo AP, Santos CC, Fonseca DC. Transtorno da compulsão alimentar periódica. Rev Psiq Clin. 2004; 31(4):170-2

31. Gluck ME, Geliebter A, Satov T. Night eating syndrome is associated with depression, low self-esteem, reduced daytime hunger, and less weight loss in obese outpatients. Obes Res. 2001; 9(4):264-7.

32. Duchesne M, Almeida PEM. Terapia cognitivocomportamental dos transtornos alimentares. Rev Bras Psiquiatr 2002; 24(Supl 3):49-53.

33. Teufel M, Becker S, Rieber N, Stephan K, Zipfel S. Psychotherapy and obesity: strategies, challenges and possibilities. Nervenartz. 2011; 82(9):1133-9.

34. Taylor VH, Stonehocker B, Steele M, Sharma AM. An overview of treatments for obesity in a population with mental illness. Can J Psychiatry. 2012; 57(1):13-20.

35. Fairburn CG, Cooper Z, Shafran R, Wilson GT. Transtorno da alimentação: um protocolo transdiagnóstico. In: Barlow DH, editor. Manual clínico dos transtornos psicológicos: tratamento passo a passo. 4th ed. Porto Alegre: Artmed; 2009. p. 577-614.

36. Cahn SC, McFillin RK. Eating disorders. In: Di Tomasso RA, Golden BA, Morris HJ, editors. Handbook of cognitive-behavioral approaches in primary care. New York: Springer Publishing Company; 2010. p. 501-23.

37. Costa MB, Melnik T. Effectiveness of psychosocial interventions in eating disorders: an overview of Cochrane systematic reviews. Einstein. 2016; 14(2):235-77.

38. Shaw KA, O'Rourke P, Del Mar C, Kenardy J. Psychological interventions for overweight or obesity. Cochrane Database Syst Rev. 2005; (2):CD003818.

39. Beck AT. Thinking and depression: II: theory and 
therapy. Arch Gen Psychiatry. 1964; 10:561-71.

40. Beck JS. Pense magro por toda a vida. Porto Alegre: Artes Médicas; 2011.

41. Luz FQ, Oliveira MS. Terapia cognitivo-comportamental da obesidade: uma revisão da literatura. Aletheia. 2013; (40):159-73.

42. Cortez MC, Araújo EA, Ribeiro MV. Transtorno de compulsão alimentar periódico e obesidade. Arq Cat Med. 2011; 40(1):94-102.

43. Fairburn CG, Marcus MD, Wilson GT. Cognitivebehavioral therapy for binge eating and bulimia nervosa: a comprehensive treatment manual. In: Fairburn CG, Wilson GT, editors. Binge eating: nature, assessment and treatment. New York: Guilford Press; 1993. p. 371- 404.

44. Devlin MJ, Fischer SE. Treatment of binge eating disorder. In: Wonderlich S, Mitchell J, de Zwaan M, Steiger H, editors. Eating disorders review. Part 1. Oxford: Radcliffe Publishing; 2005. p. 27- 41.

45. Duchesne M, Appolinário JC, Rangé BP, Freitas S, Papelbaum M, Coutinho W. Evidências sobre a terapia cognitivo-comportamental no tratamento de obesos com transtorno da compulsão alimentar periódica. Rev Psiquiatr. 2007; 29(1):80-92.

46. Abreu CN, Roso ME, organizadores. Psicoterapias cognitiva e construtivista: novas fronteiras da prática clínica. Porto Alegre: Artmed; 2003.

47. Vasques F, Martins FC, Azevedo AP. Aspectos psiquiátricos do tratamento da obesidade. Rev Psiquiatr Clin. 2004; 31(4):195-8

48. Devlin MJ. Binge-eating disorder and obesity: a combined treatment approach. Psychiatr Clin North Am. 2001; 24(2):325-35.

\section{CONFLITO DE INTERESSES}

Os autores declaram não haver conflitos de interesse.

\section{AUTOR PARA CORRESPONDÊNCIA}

Ellerson Gaetti jardim Júnior

egaettij@foa.unesp.br

Submetido em 15/11/2017

Aceito em 09/01/2018 